\title{
LIFTINGS AND THE PROPERTY OF BAIRE IN LOCALLY COMPACT GROUPS
}

\author{
MAXIM R. BURKE
}

(Communicated by William J. Davis)

\begin{abstract}
For each locally compact group $G$ with Haar measure $\mu$, we obtain the following results. The first is a version for group quotients of a classical result of Kuratowski and Ulam on first category subsets of the plane. The second is a strengthening of a theorem of Kupka and Prikry; we obtain it by a much simpler technique, building on work of Talagrand and Losert.
\end{abstract}

Theorem 1. If $G$ is $\sigma$-compact, $H \subseteq G$ is a closed normal subgroup, and $\pi: G \rightarrow G / H$ is the usual projection, then for each first category set $A \subseteq G$, there is a first category set $E \subseteq G / H$ such that for each $y \in(G / H)-E$, $A \cap \pi^{-1}(y)$ is a first category set relative to $\pi^{-1}(y)$.

Theorem 2. If $G$ is not discrete, then there is a Borel set $E \subseteq G$ such that for any translation-invariant lifting $\rho$ for $(G, \mu), \rho(E)$ is not universally measurable and does not have the Baire property.

\section{INTRODUCTION}

A lifting for a measure space $(X, \mu)$ is a Boolean homomorphism $\rho$ from the algebra $\Sigma$ of measurable sets into itself, whose kernel is the ideal of sets of measure zero, and for which $\rho(E) \Delta E$ has measure zero for each $E \in \Sigma$. If $X$ is a topological space and the domain of $\mu$ includes the open sets, then $\rho$ is called a strong lifting if $U \subseteq \rho(U)$ for each open set $U$. An equivalent way of giving a lifting is to give a lifting for $\mathscr{L}^{\infty}(X, \mu)$, i.e., an algebra homomorphism $\rho$ from $\mathscr{L}^{\infty}(X, \mu)$ into itself for which $\rho(f)=f$ a.e. whenever $f \in \mathscr{L}^{\infty}(X, \mu)$. See [12] for the equivalence.

Every complete $\sigma$-finite measure space has a lifting [M], every locally compact group has a translation-invariant lifting for its Haar measure (i.e., $\rho(g E)=$ $g \rho(E)$ for each element $g$ of the group and for each measurable set $E$ ), and every translation-invariant lifting is strong [I1]. [For each group concept that has a 'left' and a 'right' version (e.g., translation, coset, Haar measure), the 'left' version is intended in this paper.]

The constructions of liftings are not effective. The necessity of their noneffectiveness has been demonstrated in several ways. The existence of a lifting $\rho$

Received by the editors July 30, 1991.

1991 Mathematics Subject Classification. Primary 46G15, 28A51, 28C10; Secondary 54H05, 28A05.

Research supported by an NSERC operating grant. 
for Lebesgue measure on $\mathbf{R}$ implies in $Z F+D C$ the existence of a nonprincipal ultrafilter on $\mathbf{N}$. [If $x \in \mathbf{R}$ is any point not in $\rho(I) \Delta I$ for any rational interval $I$, then

$$
\mathscr{F}=\left\{A \subseteq \mathbf{N}: x \in \rho\left(\bigcup\left\{\left(\frac{-1}{(n+1)}, \frac{-1}{n}\right) \cup\left(\frac{1}{(n+1)}, \frac{1}{n}\right): n \in A\right\}\right)\right\}
$$

is a nonprincipal ultrafilter on $\mathbf{N}$.] Hence the axiom of choice is needed to produce a lifting (see [So]). (See also [C, Chapters 5 and 6].) Shelah showed that it is relatively consistent with $Z F C$ that there are no Borel liftings for Lebesgue measure on $\mathbf{R}$ [S]. [Whenever a property of sets is used to describe a lifting, we mean that the sets in the range of the lifting have this property. Thus a Borel lifting is one for which $\rho(E)$ is a Borel set for each measurable set $E$.] This result has been extended in [J, BJ] (respectively in [BS]) to show that it is relatively consistent with $Z F C$ that there are no projective (resp. Borel) liftings for the Haar measure in any power $2^{\kappa}=\{0,1\}^{\kappa}$ of the two element group.

The aspect of noneffectiveness that will interest us here was first discovered by Johnson in [J]. He showed in $Z F C$ that there can be no translation-invariant Borel lifting for the Haar measure on $\mathbf{R} / \mathbf{Z}$. This result was generalized by Talagrand who proved the following theorem.

0.1. Theorem [T]. For each locally compact abelian group $G$ that is not discrete, there is a Borel set $E$ and a compact set $L$ such that for any translationinvariant lifting $\rho, \rho(E) \cap L$ is not universally measurable and does not have the property of Baire relative to $L$.

(The assumption that $G$ is abelian can be dispensed with. This, among other things, was shown by Losert in [L], subsequent to the appearance of Theorem 0.2.)

Kupka and Prikry obtained the following result. (See [KP, Theorem 3.2 and its proof].)

0.2. Theorem [KP]. For each locally compact group $G$ that is not discrete, there is an $(\mathbf{R} / \mathbf{Z})$-valued function $f$ such that for any translation-invariant lifting $\rho$, $\rho(f)$ does not have the property of Baire.

The proof of this theorem is fairly elaborate. The function $f$ is not easy to describe. By contrast, in Talagrand's approach, the obstacles, $E$ and $L$, to having a translation-invariant Borel lifting are easy to describe.

(For example, for $G=\{0,1\}^{\omega}$ with the usual Haar measure, we have $L=$ $\{x \in G: x(n)=0$ for every even $n\}$ and $E=\{x \in G$ : for some even $n$, $x(n)=1$, and for the least such $n,|\{j<n: x(j)=0\}|$ is even $\}$.)

The conclusions of Theorems 0.1 and 0.2 , however, are somewhat different. On the one hand, it is not clear whether the function $f$ in Theorem 0.2 is universally measurable, nor whether it can be taken to be a characteristic function (and still be independent of $\rho$ ). On the other hand, the set $L$ of Theorem 0.1 is typically nowhere dense, so that $\rho(E)$ may very well have the property of Baire. (Indeed, in the example given above for $\{0,1\}^{\omega}, \rho(E)$ necessarily has the property of Baire since $E$ is open and $\rho$ is strong.)

In this paper we provide (in Theorem 2.1) a generalization of Theorems 0.1 and 0.2 , which gives a strong obstacle to the existence of a translationinvariant lifting with nice descriptive properties, using the elegant approach of 
[T]. In order to arrange that $\rho(E)$ does not have the property of Baire, some new ideas are needed. Among other things, we will give a version for group quotients (Theorem 1.2) of a well-known theorem of Kuratowski and Ulam on first category subsets of the plane [KU] (or see [O, Theorem 15.1]). It provides, for first category sets, the information that Weil's formula [H, Theorem 63G] provides for sets of measure zero.

\section{First CATEgory SETS AND THE PROPERTY OF BAIRE}

1.1. Lemma. If $X$ and $Y$ are topological spaces, $X$ is second countable, and $\pi: X \rightarrow Y$ is a continuous open surjection, then for every nowhere dense (resp. first category) set $A \subseteq X$, there is a first category set $E \subseteq Y$ such that for all $y \in Y-E, A \cap \pi^{-1}(y)$ is nowhere dense (resp. first category) relative to $\pi^{-1}(y)$.

Proof. It suffices to prove the lemma when $A$ is closed nowhere dense. Suppose $Y_{1}=\left\{y \in Y: \pi^{-1}(y) \cap A\right.$ is somewhere dense in $\left.\pi^{-1}(y)\right\}$ is second category. For each $y \in Y_{1}$, choose a basic open set $U(y)$ (from some fixed countable base for the topology of $X$ ) such that $\varnothing \neq U(y) \cap \pi^{-1}(y) \subseteq A$. Let $Y_{2} \subseteq Y_{1}$ be a set of second category for which there is an open set $U$ such that $U(y)=U$ for all $y \in Y_{2}$. But now $Y_{2} \subseteq \pi(U)-\pi(U-A)$, which is a contradiction since $\pi(U-A)$ is a dense open subset of $\pi(U)$.

1.2. Theorem. Let $G$ be a $\sigma$-compact locally compact group, $H \subseteq G$ a closed normal subgroup, and $\pi_{H}: G \rightarrow G / H$ the usual projection. Then for every nowhere dense (resp. first category) set $A \subseteq G$, there is a first category set $E \subseteq$ $G / H$ such that for all $y \in(G / H)-E, A \cap \pi_{H}^{-1}(y)$ is nowhere dense (resp. first category) relative to $\pi_{H}^{-1}(y)$.

Proof. Again we need only consider the case where $A$ is nowhere dense. Furthermore, since $G$ is completely regular and satisfies the countable chain condition [the Haar measure on $G$ is $\sigma$-finite], we may take $A$ to be a closed Baire set. By [H, Theorem $64 \mathrm{G}$ ], there is a compact normal subgroup $K$ of $G$ such that $\pi_{K}^{-1} \pi_{K}(A)=A$ where $\pi_{K}: G \rightarrow G / K$ is the usual projection. Let us denote by $\bar{\pi}_{H}$ and $\bar{\pi}_{K}$ the projections $G / H \rightarrow G / H K$ and $G / K \rightarrow G / H K$, respectively (so that $\bar{\pi}_{H} \pi_{H}=\bar{\pi}_{K} \pi_{K}$ ).

By Lemma 1.1 , there is a first category $F_{\sigma}$ set $E \subseteq G / H K$ such that for each $y \in(G / H K)-E, \pi_{K}(A) \cap \bar{\pi}_{K}^{-1}(y)$ is nowhere dense in $\bar{\pi}_{K}^{-1}(y)$. Since $\bar{\pi}_{H}$ is open, $\bar{\pi}_{H}^{-1}(E)$ is a first category $F_{\sigma}$ in $G / H$. We claim that for each $x \in(G / H)-\bar{\pi}_{H}^{-1}(E), A \cap \pi_{H}^{-1}(x)$ is nowhere dense in $\pi_{H}^{-1}(x)$.

Suppose not, and let $x$ be a counterexample. Let $y=\bar{\pi}_{H}(x) \notin E$. Fix $\alpha \in \pi_{H}^{-1}(x)$ (so $\pi_{H}^{-1}(x)=\alpha H$ ). In $H$, let $V$ be a nonempty open set such that

$$
\alpha V \subseteq A \cap \pi_{H}^{-1}(x)=A \cap \alpha H .
$$

Then

$$
\pi_{K}(\alpha V) \subseteq \pi_{K}(A) \cap \bar{\pi}_{K}^{-1}(y) .
$$

We shall have a contradiction if we can show that $\pi_{K}(\alpha V)$ is open relative to $\bar{\pi}_{K}^{-1}(y)$.

Note that, since $K$ is compact, $V K$ is open relative to $H K$. [ $V K=$ $V(H \cap K) K=V^{\prime} K$ where $V^{\prime}=V(H \cap K)$ is open in $H$. Now $\left(H-V^{\prime}\right) K$ is closed in $H K$ [HR, Theorem 4.4] and $H K$ is the disjoint union of $V^{\prime} K$ 
and $\left(H-V^{\prime}\right) K$ (since $\left.V^{\prime}=V^{\prime}(H \cap K)\right)$.] Thus $\alpha V K$ is open relative to $\alpha H K=\pi_{H}^{-1}(x) K=\pi_{H}^{-1} \bar{\pi}_{H}^{-1}(y)=\pi_{K}^{-1} \bar{\pi}_{K}^{-1}(y)$. Thus $\pi_{K}(\alpha V)=\pi_{K}(\alpha V K)=$ $\pi_{K}\left(\pi_{K}^{-1} \pi_{K}(\alpha V)\right)$ is open relative to $\pi_{K}\left(\pi_{K}^{-1} \bar{\pi}_{K}^{-1}(y)\right)=\bar{\pi}_{K}^{-1}(y)$, which is the desired contradiction.

Kuratowski and Ulam give a counterexample to Theorem 1.2 when $G$ is not $\sigma$-compact; see [KU]. To see that the group-theoretic assumptions made on $G$ cannot be removed, consider the following example.

1.3. Example. Let $X$ be the Stone space of the measure algebra of $[0,1]$ and let $Y=[0,1]$, so that $Y \times X$ is a compact $\mathrm{T}_{2}$ Radon probability space (with Lebesgue measure on $Y$, the induced measure on $X$, and the usual product measure on $Y \times X)$ that is self-supporting (= each nonvoid open set has positive measure; the point is that this is a strong chain condition that is also possessed by any $\sigma$-compact locally compact group). Let $\pi: Y \times X \rightarrow Y$ be the projection map. Then there is a first category set $A \subseteq Y \times X$ such that for each $y \in Y$, $A \cap \pi^{-1}(y)$ is a nonvoid open set relative to $\pi^{-1}(y)$.

Construction. Since any perfect Polish space contains a residual copy of the irrationals, we may take for $Y$ any perfect Polish space. Let $\mathscr{K}$ be the set of all compact subsets of $[0,1]$ with the exponential topology. (See $[\mathrm{K}, \S \S 17,21]$ for the basic properties of this topology.) $\mathscr{K}$ is a compact metrizable space. It is convenient here to use $Y=\left\{K \in \mathscr{K}: \mu K \geq \frac{1}{2}\right\}$, which is a closed perfect subspace of $\mathscr{K}$. Let $A \subseteq Y \times X$ be given by

$$
A=\bigcup\left\{\{K\} \times K^{*}: K \in Y\right\}
$$

where $K^{*}$ is the set of all ultrafilters in $X$ that contain the equivalence class of $K$. Then the vertical sections of $A$ are clearly as desired. It remains to see that $A$ is nowhere dense in $Y \times X$.

Let $U \times V$ be a nonvoid basic open set in $Y \times X$. Choose open sets $W$ and $W_{i} \subseteq W, i=1,2, \ldots, n$, such that $U=\left\{K \in Y: K \subseteq W\right.$ and $K \cap W_{i} \neq \varnothing$ for all $i$, and choose a set $M$ of positive measure such that $V=M^{*}$. Since $U \neq \varnothing$, we have $\mu W>\frac{1}{2}$. Fix any density point of $M$, and choose a small enough closed interval centered on this density point so that $\mu(W-I)>\frac{1}{2}$ and $W_{i}-I \neq \varnothing$ for all $i$. Let

$$
U^{\prime}=\left\{K \in Y: K \subseteq W-I \text { and } K \cap\left(W_{i}-I\right) \neq \varnothing \text { for all } i\right\},
$$

and let $V^{\prime}=(M \cap I)^{*}$. Then $U^{\prime} \times V^{\prime}$ is a nonvoid open subset of $U \times V$ and $\left(U^{\prime} \times V^{\prime}\right) \cap A=\varnothing$.

1.4. Remark. In the next section we will use only the special case of Theorem 1.2 in which $A=\pi^{-1}(\bar{A})$ for some $\bar{A} \subseteq G / H$. This special case holds in much greater generality, as the next theorem shows. Its proof is very similar to that of Theorem 3(9) in [W] (a result which was brought to our attention by S. Todorcevic) and we leave it to the reader. See [W] for the definition of a weakly $\alpha$-favorable space. We note here only that locally compact spaces are weakly $\alpha$-favorable.

1.5. Theorem. If $X$ is weakly $\alpha$-favorable, $\pi: X \rightarrow Y$ is an open continuous surjection, and $\bar{A} \subseteq Y$ is second category in $Y$, then $\pi^{-1}(\bar{A})$ is second category in $X$. 


\section{TRANSLATION-INVARIANT LIFTINGS}

2.1. Theorem. Let $G$ be a nondiscrete locally compact group with Haar measure $\mu$. Then there is a Borel set $E \subseteq G$ such that for any translation-invariant lifting $\rho$ for $(G, \mu), \rho(E)$ is not universally measurable and does not have the property of Baire.

Proof. As in [KP, first two paragraphs of the proof of Theorem 3.2], we may assume that $G$ is $\sigma$-compact, and we may find a compact normal subgroup $K \subseteq G$ such that $G / K$ is second countable and not discrete. Let $\pi: G \rightarrow G / K$ be the projection map. It will be enough to prove the theorem for $G / K$. For suppose that we have found a set $E \subseteq G / K$ as in the conclusion of the theorem. Then $\pi^{-1}(E)$ will work for $G$. The reason for this is as follows. Suppose $\rho$ is a translation-invariant lifting for $(G, \mu)$. Then we can define a lifting $\bar{\rho}$ for $G / K$ as follows. For each measurable set $S \subseteq G / K, \pi^{-1}(S)$ is invariant under $K$ (by translations both on the left and on the right since $K$ is normal). By the translation-invariance of $\rho, \rho\left(\pi^{-1}(S)\right)$ is also invariant under $K$. Let $\bar{\rho}(S)=$ $\rho\left(\pi^{-1}(S)\right)$. The Haar measure $\mu$ on $G$ is carried by $\pi$ to the Haar measure $\bar{\mu}$ on $G / K$ (because $K$ is compact; see [H, Theorem $63 \mathrm{C}]$ ). By inner regularity of these measures, it is easy to see that $\bar{\rho}(S)$ is measurable and belongs to the equivalence class of $S$. It is also easy to see that $\bar{\rho}$ is a Boolean homomorphism and is translation-invariant. Suppose now that $\rho\left(\pi^{-1}(E)\right)$ has the property of Baire. Let $U$ be the unique regular open set such that $F=U \Delta \rho\left(\pi^{-1}(E)\right)$ is first category (see [O, Theorem 4.6]). Since $\pi^{-1}(E)$ is invariant under $K$, so are $\rho\left(\pi^{-1}(E)\right), U$, and $F$. Thus $\bar{\rho}(E)=\pi(U) \Delta \pi(F)$. Now $\pi(U)$ is open and $\pi(F)$ is first category by Theorem 1.2. Hence $\bar{\rho}(E)$ has the property of Baire-a contradiction.

To see that $\rho\left(\pi^{-1}(E)\right)$ is not universally measurable, fix a Radon measure $\bar{\lambda}$ on $G / K$ for which $\bar{\rho}(E)$ is not measurable. Let $\lambda$ be a Radon measure on $G$ such that $\pi \lambda=\bar{\lambda}$. It is easy to show that if $\rho\left(\pi^{-1}(E)\right)=\pi^{-1}(\bar{\rho}(E))$ is $\lambda$-measurable, then $\bar{\rho}(E)$ is $\bar{\lambda}$-measurable.

Henceforth we assume that the group $G$ is separable and metrizable. Fix a left-invariant metric on $G$ [HR, Theorem 8.3]. (The word 'diameter' in what follows refers to this nameless metric.)

We shall define open sets $V_{r}(s)$, where for some $k<\omega, r \in 2^{k}$ and $s \in \omega^{k}$, such that $V_{\varnothing}(\varnothing)=G$ and for each $k \neq 0$ and for each $r \in 2^{k}$ and $s \in \omega^{k}$, the following conditions are satisfied.

(1) $V_{r}(s)$ has diameter at most $1 / k$.

(2) The sets $V_{r^{-j}}\left(s^{\sim} n\right), j<2, n<\omega$, are pairwise disjoint and their closures are compact subsets of $V_{r}(s)$.

(3) $V_{r}(s)-\bigcup_{j<2} \bigcup_{n<\omega} V_{r^{-} j}\left(s^{\frown} n\right)$ is nowhere dense.

(4) $\bigcup_{r \in 2^{k}, s \in \omega^{k}} V_{r}(s)$ has measure at most $1 / k$.

We will also define elements $x(s) \in G$ such that the following condition holds. Let us write $\overline{0}$ for the zero element in $2^{k}$ (letting the context determine $k$ ).

(5) For each nonzero $k<\omega$, and for all $i<k, s \in \omega^{k}, r \in 2^{k}$, if $r \neq \overline{0}$ and $i_{1}<\cdots<i_{l}<k$ are the numbers $i$ such that $r(i)=1$, then $p(r, s) V_{\overline{0}}(s)=V_{r}(s)$ where $p(r, s)=x\left(s \mid\left(i_{1}+1\right)\right) x\left(s \mid\left(i_{2}+1\right)\right) \cdots x\left(s \mid\left(i_{l}+1\right)\right)$.

The initial step $k=1$. Let $\left\{W_{n}: n<\omega\right\}$ list a base for the topology of $G$. Inductively choose elements $x(n) \in G$ and pairs of open sets 
$V_{0}(n), V_{1}(n)=x(n) V_{0}(n)$ with compact closures such that $V_{0}(n)$ has diameter at most 1 and has measure at most $1 / 2^{n+2}$, the sets $V_{0}(n), V_{1}(n) \quad(n<\omega)$ are pairwise disjoint, and $\bigcup_{k<n} V_{0}(k) \cup V_{1}(k)$ has nonvoid intersection with $W_{n}$ but is not dense in $G$. This is straightforward. Use the fact that $G$ is not discrete and hence perfect, as well as the fact that the points of $G$ have measure zero ( $G$ is locally compact, compact sets have finite measure, and $G$ is not discrete). It is clear that all the conditions are now satisfied for $k=1$.

The inductive step to $k+1$. Fix a collection $\left\{\varepsilon(s): s \in \omega^{k+1}\right\}$ of positive real numbers such that

$$
\sum_{s \in \omega^{k+1}} \varepsilon(s) 2^{k+1} \leq \frac{1}{k+1} .
$$

For each $s \in \omega^{k}$, list a base $\left\{W_{n}(s): n<\omega\right\}$ for the topology of $V_{\overline{0}}(s)$ and inductively choose elements $x\left(s^{\frown} n\right) \in G$ and pairs of open sets $V_{\overline{0}}{ }_{0}\left(s^{\wedge} n\right)$,

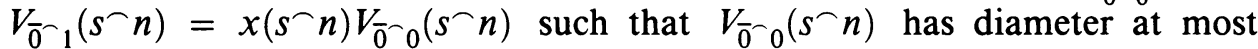
$1 /(k+1)$ and has measure at most $\varepsilon\left(s^{\urcorner} n\right)$, the sets $V_{\overline{0}^{\wedge} 0}\left(s^{\frown} n\right)$, $V_{\overline{0}-1}\left(s^{\frown} n\right) \quad(n<\omega)$ are pairwise disjoint and have compact closures contained in $V_{\overline{0}}(s)$, and $\left.\bigcup_{k \leq n} V_{\overline{0}}{ }^{\prime}(s \neg k) \cup V_{\overline{0}-1}(s\urcorner k\right)$ has nonvoid intersection with $W_{n}(s)$ but is not dense in $V_{\overline{0}}(s)$.

For other $r \in 2^{k}$, let

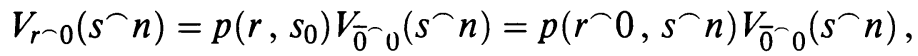

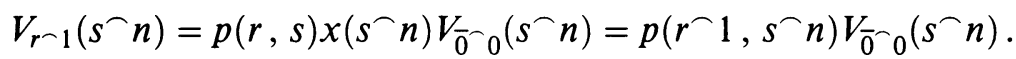

Since translation by $p(r, s)$ is a measure-preserving isometry between $V_{\overline{0}}(s)$ and $V_{r}(s)$, conditions (1), (2), and (3) are satisfied. Condition (4) holds by the choice of the $\varepsilon(s)$ 's, and condition (5) holds by definition of the $V_{r}(s)$ 's.

Let $U_{k}=\bigcup\left\{V_{r}(s): r \in 2^{k}, s \in \omega^{k}\right\}$. This is a dense open set of measure at most $1 / k$. Thus $\bigcap_{k} U_{k}$ is a dense $G_{\delta}$ set of measure zero.

2.2. Remark. By condition (1), the sets $V_{r}(s)$ that contain a given point $x \in$ $\bigcap_{k} U_{k}$ form a base at $x$.

Now define $E \subseteq G$ as follows.

For each $k \neq 0$ and for each $s \in \omega^{k}$, partition $V_{\overline{0}}(s)-U_{k+1}$ into two disjoint sets of equal measure, $A_{\overline{0}}(s)$ and $B_{\overline{0}}(s)$. For $r \in 2^{k}$, let $l(r)=\mid\{i<k: r(i)=$ $1\} \mid$. Define

$$
A_{r}(s)= \begin{cases}p(r, s) A_{\overline{0}}(s), & l(r) \text { even }, \\ p(r, s) B_{\overline{0}}(s), & l(r) \text { odd },\end{cases}
$$

so that $\left\{A_{r}(s), B_{r}(s)\right\}$ is a partition of $V_{r}(s)-U_{k+1}$ into two sets of equal measure. (Note that $p(r, s)\left(V_{\overline{0}}(s)-U_{k+1}\right)=V_{r}(s)-U_{k+1}$.)

Let $E=\bigcup_{r, s} A_{r}(s)$.

Suppose that for some translation-invariant lifting $\rho, \rho(E)$ has the property of Baire. Then one of $\rho(E), \rho\left(E^{c}\right)$ contains a nonvoid open set modulo a first category set, say the former.

Then $\rho(E) \cap \bigcap_{k} U_{k}$ contains a nonvoid open set modulo a first category set, and by Remark 2.2, there is a $k \neq 0$ and there are sequences $r \in 2^{k}$ and $s \in \omega^{k}$ such that $V_{r}(\bar{s}) \subseteq \rho(E)$ modulo a first category set.

Let $s=\bar{s}^{\frown} n$ be any extension of $\bar{s}$. 
Choose $y \in\left(V_{r-0}(s) \cap \rho(E)\right)$ such that $p\left(r^{-1}, s\right) p\left(r^{-0}, s\right)^{-1} y \in V_{r-1}(s) \cap$ $\rho(E)$. For each $i \geq k+1$, we have

$$
\begin{gathered}
p\left(r^{\frown} 1, s\right) p(r \frown 0, s)^{-1}\left(E \cap V_{r-0}(s) \cap\left(U_{i}-U_{i+1}\right)\right) \\
\quad=\left[V_{r-1}(s)-\left(E \cap V_{r-1}(s)\right)\right] \cap\left(U_{i}-U_{i+1}\right) .
\end{gathered}
$$

[For each $a \in 2^{i}, b \in \omega^{i}$ such that $a \supseteq r^{\frown} 0, b \supseteq s$, let $\bar{a}$ denote the element of $2^{k}$ that agrees with $a$ everywhere except at $k$ (and $\bar{a}(k)=1$ ). We have $p(\bar{a}, b) p(a, b)^{-1}=p\left(r^{-1}, s\right) p\left(r^{-} 0, s\right)^{-1}$. Also one of $l(a), l(\bar{a})$ is even, and the other one is odd. Hence

$$
p\left(r^{\frown} 1, s\right) p\left(r^{\frown} 0, s\right)^{-1}\left(E \cap V_{a}(b)-U_{i+1}\right)=\left[V_{\bar{a}}(b)-\left(E \cap V_{\bar{a}}(b)\right)\right]-U_{i+1} .
$$

Taking the union over all such $a, b$ gives the desired equality.]

Now taking the union over all $i$ we get

$$
p\left(r^{\frown} 1, s\right) p\left(r^{\frown} 0, s\right)^{-1}\left(E \cap V_{r \smile 0}(s)\right)=V_{r-1}(s)-\left(E \cap V_{r-1}(s)\right)-\bigcap_{k} U_{k} .
$$

Applying $\rho$ to this equality we see that $\rho\left(V_{r-1}(s)\right)$ is the disjoint union of

$$
p\left(r^{\frown} 1, s\right) p\left(r^{-0} 0, s\right)^{-1} \rho\left(E \cap V_{r-0}(s)\right) \text { and } \rho\left(E \cap V_{r-1}(s)\right) .
$$

However, this is impossible since the choice of $y$ guarantees that

$$
p\left(r^{\frown} 1, s\right) p\left(r^{\frown} 0, s\right)^{-1} y
$$

belongs to both of these sets. (We have $p\left(r^{\frown 1, s)} p\left(r^{\frown} 0, s\right)^{-1} y \in \rho\left(V_{r^{-1}}(s)\right)\right.$ since $\rho$ is a strong lifting.)

To see that $\rho(E)$ is not universally measurable, define $h: 2^{\omega} \rightarrow G$ by $h(f)=$ the unique member of $\bigcap_{k} V_{f \mid k}(\overline{0})$. Conditions (1) and (2) ensure that $h$ is a homeomorphism onto its range. If we let $\lambda$ be the image under $h$ of the usual Haar measure on $2^{\omega}$, then as in [T], $\rho(E)$ is not $\lambda$-measurable.

\section{ACKNOWLEDGMENT}

The author thanks the Department of Mathematics of the University of Toronto for its hospitality while this paper was being written.

\section{REFERENCES}

[BJ] M. R. Burke and J. Just, Liftings for Haar measure on $\{0,1\}^{\kappa}$, Israel J. Math. 73 (1991), 33-44.

[BS] M. R. Burke and S. Shelah, Linear liftings for non-complete probability spaces, Israel J. Math. (to appear).

[C] J. P. R. Christensen, Topology and Borel structure, North-Holland, Amsterdam, 1974.

[H] P. R. Halmos, Measure theory, Springer-Verlag, New York, 1974.

[HR] E. Hewitt and R. A. Ross, Abstract harmonic analysis. I, 2nd ed., Springer-Verlag, Berlin, 1979.

[I1] A. Ionescu-Tulcea and C. Ionescu-Tulcea, On the existence of a lifting commuting with the left translations of an arbitrary locally compact group, Proc. Fifth Berkeley Sympos. Math. Statist. and Probab. (Berkeley Calif. 1965/66), vol. II; Contributions to Probab. Theory, Part I, Univ. of Calif. Press, Berkeley, CA, 1967, pp. 63-97. , Topics in the theory of liftings, Springer-Verlag, New York, 1969. 
[J] R. A. Johnson, Strong liftings which are not Borel liftings, Proc. Amer. Math. Soc. 80 (1980), 234-236.

[Ju] W. Just, A modification of Shelah's oracle-cc with applications, Trans. Amer. Math. Soc. 329 (1992), 325-356.

[K] K. Kuratowski, Topology, vol. I, Academic Press, New York, 1966.

[KP] J. Kupka and P. Prikry, Translation-invariant Borel liftings hardly ever exist, Indiana Univ. Math. J. 32 (1983), 717-731.

[L] V. Losert, Some remarks on invariant liftings, Measure Theory Oberwolfach 1983 (D. Kölzow and D. Maharam-Stone, eds.), Lecture Notes in Math., vol. 1080, Springer-Verlag, Berlin, 1984, pp. 95-110.

[M] D. Maharam, On a theorem of von Neumann, Proc. Amer. Math. Soc. 9 (1958), 987-994.

[O] J. C. Oxtoby, Measure and category, Academic Press, New York, 1971.

[S] S. Shelah, Lifting problem of the measure algebra, Israel J. Math. 45 (1983), 90-96.

[So] R. M. Solovay, A model of set theory in which every set of reals is Lebesgue measurable, Ann. of Math. (2) 92 (1970), 1-56.

[T] M. Talagrand, La pathologie des relèvements invariants, Proc. Amer. Math. Soc. 84 (1982), 379-382.

[W] H. E. White, Topological spaces that are $\alpha$-favorable for a player with perfect information, Proc. Amer. Math. Soc. 50 (1975), 477-482.

Department of Mathematics and Computer Science, University of Prince Edward Island, Charlottetown, Prince Edwards Island, Canada C1A 4P3

E-mail address: burke@upei.ca 